\title{
Develop Human Safety Mechanism for Human-Symbiotic Mobile Manipulators: Compliant Hybrid Joints
}

\author{
Zhijun Li[1], Jun Luo[2] , Shaorong Xie ${ }^{[2]}$, Jiangong Gu[1] \\ [1] Department of Mechanical and Control Engineering \\ The University of Electro-Communications, JAPAN \\ [2] School of Mechatronics Engineering and Automation \\ Shanghai University, Shanghai, China
}

\section{Introduction}

Today, robots are expected to provide various services directly to humans in environments, this situation has led to the idea of teams consisting of humans and robots working cooperatively on the same task. Various names for this type of human-robot cooperation system have emerged including human-friendly robots, personal robots, assistant robots and symbiotic robots. These robots will continue to be employed also in the 21st century to cope with the increase in the elderly and handicapped, the decrease in the birth rate and working population and will be introduced into non-industrial areas such as homes and offices to make a rich and comfortable life. Such robots as home-use robots, assistance robots, and service robots, should deal with diverse tasks (Fujie, Tani, and Hirano 1994; Kawamura and Iskarous 1994). One of the specific situations in the non-industrial areas is that the robots coexist and help humans in their life environments. The robots, therefore, must be with the capability of human-robot coexistence. They can be called "humansymbiotic robots" (HSRs). There are various problems to be solved to develop the HSRs. Working and moving among humans requires special concerns on the safety issues, the safety of human in view of an unexpected collision should be assured. A HSR should weight not significantly more than a human, but mechanical compliance of the surface and joints is also a necessity.

In the past two decades, many researchers have studied to reduce in advance the Of unexpected collision accidents between an industrial robot and a human operator by isolating robots in work cells that automatically shut down if a person enters; visuals (signs, flashing lights), and audio devices would indicate conditions by the operation $\Omega$ and vision/sound alarms, and so on. Because these accident precautions approaches are difficult to assure human safety in human-robot environments in which an interaction between the person and the robot is presupposed, we have to consider other ways to deal with human-robot collisions and such contacts can take place anywhere on the body ๊ of the robot. Therefore, the HSR should be constructed on the basis of a new philosophy from that of past robots. Lim and Tanie (2000) proposed the HSR must be constructed for 
everybody use, with simple structure like home electronic products, human-like mobility, human-like compliance, and with the human-friendly user interface. In addition, the HSR requires a robustness and compliance to perform a human-robot cooperative task.

The novelty human safety mechanisms, therefore, should be designed and introduced into the HSR, which are able to cope with the problem that the produced impact/collision forces are caused by human-robot unconscious contacts. In this chapter, we focus on collision force suppression and develop a simple, low-cost, and effective physical mechanism using complaint hybrid joints for the human-symbiotic mobile manipulators. During expected/unexpected collisions with their environments, the hybrid joints will passively deform to reduce the produced collision force. Moreover, we propose the collision-tolerant recovery controls to realize the desired task despite the unexpected collisions. In this chapter, we also examine the control method through simulations and experiments.

\subsection{Related Research}

Making the robot human-like compliance is one good way of enhancing safety. There exist two general strategies to realize robot compliance: active compliance, and passive compliance. The active compliance is provided with a sensor feedback to achieve either a control of interaction forces or a task-specific compliance of the robot. The passive compliance is realized by using passive deformable devices attached to the robot body.

\subsubsection{Active Compliance}

The active compliance methods may be divided into force control and impedance control. Given a detailed environment description, a widely adopted method is hybrid position/force control. The 'hybrid' characterization should be the simultaneous control of either position or force in a given directions, not both. The task space is partitioned into two orthogonal subspaces. The scheme allows adjustment of position and force dynamics independently (Raibert \& Craig 1981; Wedel \& Saridis 1988; Anderson \& Spong 1987; Schutter \& Van Brussel 1988). On the other hand, impedance control is to enforce an adjustable mechanical impedance relationship between the force and the position error. Proper adjustment of the impedance parameters ensures bounded contact forces. The primary merit of impedance control is that it establishes adjustable balanced behavior of the system between position errors and external force (Salisbury 1980; Whitney 1977; Kazerooni \& Waibel 1988; Goldenberg 1987).

\subsubsection{Passive Compliance}

The most past passive compliance methods were based on the robot's structural compliance using special devices such as springs and dampers. Whitney (1982) described the usefulness of remote center compliance devices for peg-in-hole insertion tasks. Goswami and Peshkin (1993) described the use of hydraulic cylinders to provide a passive wrist with programmable accommodation, and they showed how accommodation and damping matrices transform between task-space and jointspace of passive redundant manipulators. Cutkosky and Wright (1986) extended the number of compliance centers available from a passive wrist by adding pressure-controlled and fluid-filled bladders. Mills (1990) used hybrid actuators consisting of a DC servo motor paired with a pneumatic bladder actuator to vary manipulator stiffness. Lindsay, Sinha, and Paul (1993) used rubber elements in the robot wrist to reduce the effect of impacts. Laurin-Kovitz, Colgate, and 
Carnes (1991) described the programmable passive impedance control using antagonistic nonlinear springs and binary dampers and showed that the impedance of a robot might be controlled by incorporating programmable mechanical elements into the robot's driving system. Other researchers have studied collision force attenuation with human safety in mind. Suita et al. (1995) and Yamada et al (1997) addressed the human-oriented design approach to developing a viscoelastic covering for the passive compliance and set up safety condition that an arm is called "safe" if the impact force is in an acceptable pain tolerance limit. Morita and Sugano $(1995,1996)$ and Morita, Shibuya, and Sugano (1998) developed the mechanical compliance adjuster of which the compliant springs are mounted in the Wendy robot arm's joints. In their studies, the combination of the safety material and the compliance control was described for the effective attenuation of collision force produced by an unexpected collision. Lim and Tanie (1999) proposed a robot with a passive trunk for human-robot coexistence. The passive trunk is composed of linear springs and dampers between a mobile part and an arm. Other different compliant passive mechanism is that such as Yoon, Kang, Kim, etc. (2003) developed the passive compliant joint composed of a magneto-rheological (MR) damper and a rotary spring, where the rotary spring gives the arm compliant property and the damper has been introduced to work as a rotary viscous damper by controlling the electric current according to the angular velocity of spring displacement. And Li (2004) proposed a novelty complaint passive mechanism, which is different from the traditional spring-damper system, the hybrid joint for holonomic mobile manipulators.

\subsection{Overview}

In this chapter, we develop a simple, low-cost, and effective physical mechanism for nonholonomic mobile manipulators, which consists of hybrid joint scheme and soft material-covering links as human safety structure against collisions. Then we propose switching control of hybrid joint, which is capable of compliantly adapting to human's motion or force by switching the hybrid joint to the active mode or the passive mode as needed depending on the requirement of a given task. Several recovering position controls of the end-effector after the collision are also presented.

This chapter is organized as follows. In Section 2, we present human safety mechanical structure for human-symbiotic robot. In Section 3, we propose the corresponding control methods for the safety structure. Section 4 describes simulation and experiment studies on collision tolerance control of mobile manipulator. The results verify the efficacies of the proposed physical mechanism and the control approach. Finally, the conclusion is presented in Section 5.

\section{Robot Structure for Human Robot Symbiosis}

HSR is assumed to share the living and working place with human beings, and therefore, is required to have the functions of human-like compliance, workability, mobility, and so on. Especially, even if a person causes a collision with the robot, he/she would not be injured. In this section, the novelty safety mechanisms are described to provide more reliable HSR. They are compliant hybrid joints and soft-covering links that can passively deform in a collision.

\subsection{A Human-Symbiotic Robot}

As the relative works of active compliance have already been discussed (see Section 1), the active control methods are also difficult to secure the high level of reliability in safety because the sensors may be saddled with essential problems involving dead angles and disturbances. In addition, when the control systems fail to function for some reason, a person involved in a collision may be 
badly injured. The active compliance control approaches use the force/torque sensors mounted on appropriate locations of the manipulator such as the manipulator wrist and joints to realize human safety by the compliance of the manipulator when the end-effector of the manipulator collides with a human. The problem in these methods, however, will relate to how the compliance parameters should be specified. Generally, the manipulator compliance is determined according to the task requirements. In case such requirements are competitive with human safety requirements, the difficulty of realizing both human safety and task performance will be produced. The active compliance methods also have the problem that the compliance of the manipulator is achieved on the basis of control software.

The passive compliance methods making the robot hardware itself compliant will be more reliable, compared with the above active compliance approaches. In general, we know the human's body consists of skeleton, soft tissue, and skin. The human's arm has passive compliant joints and is connected to his or her waist with several joints through the shoulder. In a collision, his or her joints and body should make a passive compliant motion. Lim and Tanie (2000) fully examined the human reaction to a collision with his or her environment, they thought, first, the produced impact/collision force is absorbed by the skin with a viscoelastic characteristic; second, if the collision force exceeds the tolerable limit of the elastic tissue, his or her shoulder and waist joints passively move to cope with the collision; finally, if the magnitude of the collision force exceeds the friction force developed between his or her feet and the ground, he or she unconsciously steps/slips on the ground to the direction of the collision force and reduces the collision force. They were interested in the viscoelastic compliant motion of a human's waist and the slipping and stepping motion of his or her feet, and introduced a human friendly robot. The slipping and stepping motion are important to attenuating the collision force, however, there would be time-delayed for the steps/slips on the ground compared with the hybrid joint's direct passive motion proposed in the chapter. The time delay would make the impact force very large when hardware compliance is too insufficient for the environments to improve the reliability of human safety, for example, when the relative velocity of two collision objects is fast. And, we expect the collision force as minimized as possible. Directly faster passive response/deformation would greatly decrease the interaction force. Therefore, in the chapter, we introduce the mechanisms of hybrid joints for human-robot collisions.

We develop a human-symbiotic robot (HSR) consisting of a mobile base and an arm using hybrid joints. The arm of the HSR is covered with viscoelastic covering and equipped with hybrid joints that can be switched to the active mode or the passive mode as needed depending on the requirement of the given task. The viscoelastic covering is equipped with mechanical elements such as springs and dampers. This HSR can deal with the collision between its body including the manipulator and environment. In case that the HSR or a human causes an unexpected collision with the other, its viscoelastic cover and hybrid joint passively deform according to the collision forces like human's flesh and waist. Therefore, it will not be seriously hurt due to the effective suppression of the collision forces caused by the elasticity of the body covering and the passive mobility of the hybrid joint.

\subsection{Characteristics of Hybrid Joint}

\subsubsection{Development of Hybrid Joint}

To provide a reliable robot which is safe to humans, as mentioned in Section 1. A kind of hybrid joint mechanism contributes to the reduction of too large collision forces. The hybrid joint consists of an electromagnetic clutch between the motor and the output shaft as shown 
in Fig. 1. The hybrid joint has two exchangeable modes: passive and active mode. When the clutch is released, the joint is free and switched to the passive mode, and the free link is directly controlled by the coupling characteristics of the manipulator dynamics. When the clutch is on, the link is controlled with the motor.

When the external force is applied to the ith link of the arm by a human, or due to the collision with an object in the working environment, the adjacent joint will be switched by the clutch to follow the collision force and the collision link will deform to suppress the impact force. However, the end-effector will be largely deviated from the desired trajectory and task execution ability would be deteriorated. In order to deal with this problem, we must introduce the recovery control of the arm to compensate for the deviation. Until now, there has been no method of hybrid joints to control the relation between the manipulator and the contact environment.

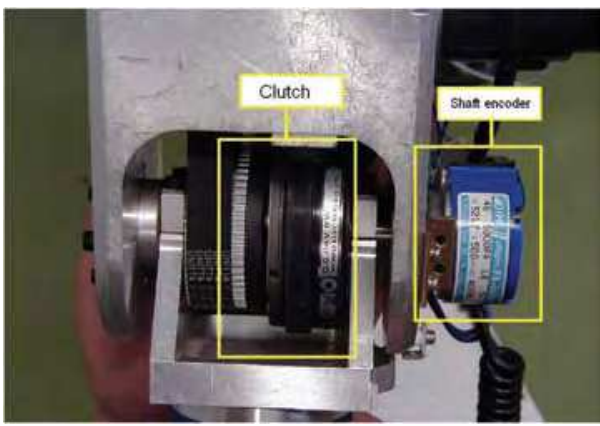

Fig. 1. A hybrid joint.

\subsubsection{Switching Logic of the Hybrid Joints for Collision Force Suppression}

Consider the hardware compliance systems being worthy of notice in collision force attenuation. To achieve the human safety for human-robot (H-R) coexistence, we need to design switching logic of the hybrid joint to avoid mental/physical damage to human. Because non-contact sensors, such as vision sensors or proximity sensors, have poor image processing capabilities as well as the ambiguity of detectable volume in proximity-sensing techniques, which makes it difficult to secure a high level of reliability in unexpected collision. Therefore, we have developed a method to the extent where H-R contact at its incipient stage can be detected by the contact sensors distributed at the link's surfaces which triggers an earlier response to the robot velocity reduction by commanding the emergency stop and the impact force attenuated by switching the joint's mode. A robot arm is constrained on a horizontal plane (Fig. 2(a)). Suppose, now, that an obstacle with constant approaching velocity causes a collision with the manipulator (Fig. 2(a)) and the impulse force $F$ is acting on somewhere of the link $i$ as Fig.2 (a), then, the static relation between the external force $F$ and joint torques is:

$$
\tau_{F}=J_{F}^{T}(q) F
$$

where the Jacobian matrix $J_{F}$ describes the differential relation between the displacement of joint space position and the position of the point $A_{F}$ in which the force $F$ is acting, it is apparent that this force does not influence directly the behavior of the manipulator beyond the link $i$, therefore, the Jacobian matrix $J_{F}$ has the form:

$$
J_{F}=\left\lfloor\begin{array}{ll}
J_{A} & 0_{m \times(n-i)}
\end{array}\right\rfloor
$$


where $J_{A}$ is a $m \times i$ matrix associated with the manipulator between the base and the point $A_{F}$ in which the force $F$ is acting, hence, (2) can be rewritten in the form:

$$
\tau_{F}=\left[\begin{array}{ll}
J_{A}^{T} F & 0_{(n-i) \times m}
\end{array}\right]^{T}
$$

When the $i$ th link make a collision with the object, to intercept the collision force transferring along the $i$ th joint to 1 st joint, the ith joint is switched to passive mode. Then the $i$ th joint force torque is equal to zero and the output force of the link to the human is zero. The impulse force can't transfer to the $(i-1)$ th joint by $J_{A}{ }^{T}$, the jth $(j<i)$ joint torque has little collision disturbance and the deform of the link attenuates the collision force.
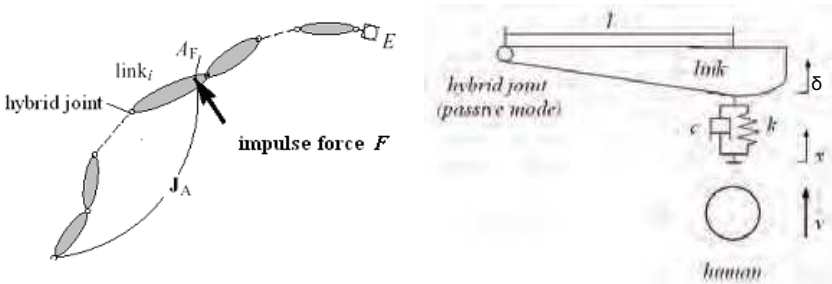

Fig. 2. (a) External force acting on the arm (left); (b) Collision model (right).

\subsubsection{Contact Model}

Beside for the hybrid joint, a viscoelastic material should be covered on the arm for absorbing the contact force in the beginning of collision. Therefore, a contact model consisting of the springdamper and the hybrid joint is studies to produce collision or contact forces during an unexpected collision or contact between a robot body or manipulator and its environment (Fig.2 (b)). Assume a robot arm is constrained on a horizontal plane, after emergency stop and switching joint, for the link using the hybrid joint contacting environment; there will be no torque present in a contact between the robot and the object. Only forces, therefore, are considered. Assume the passive joint can rotate freely, when the collision happen, the hybrid joint is released to separate both collision objects, therefore, no friction arise.

An object comes into collision/contact with a soft surface. The relationship of the collision force, the surface deformation of the robot and the contacting object is given as

$$
[M][\ddot{q}]+[C][\dot{q}]+[K][q]=0
$$

where $_{[M]}=\left[\begin{array}{cc}m & 0 \\ 0 & J\end{array}\right],[q]=\left[\begin{array}{l}x \\ \delta\end{array}\right],[C]=\left[\begin{array}{cc}c & -c l \\ -c l & c l^{2}\end{array}\right],[K]=\left[\begin{array}{cc}k & -k l \\ -k l & k l^{2}\end{array}\right], m$ is the mass of human, $J$ is the moment of inertia of the link, $\delta$ is the displacement angle of the link, $x$ is the deformation of the soft covering, $k$ is the spring coefficient , $c$ is the damp coefficient, $l$ is the distance of the collision point to the joint. The collision force $F$ can be approximately described as:

$$
F=k(x-l \delta)+c(\dot{x}-l \dot{\delta})
$$

From (5), we know the collision force is related with the distance $l$ from collision point to adjacent joint. In practice, supposing the arm is redundant enough, if the $i$ th link ( $i>1)$ itself or $l$ is shorter, in order to attenuate the impulse force, we can set a limit distance $l_{\text {lim }}$, if $l>l_{\text {lim }}$, the corresponding hybrid joint is switched, that is, the ith joint, otherwise, the jth joint $(j \leq i-1)$ is switched to meet the limit distance, the $(j+1)$ th joint is blocked. 
Develop Human Safety Mechanism for

\section{Control of Nonholonomic Mobile Underactuated Manipulator}

The advantage of mobile manipulator is to improve the flexibility of system and extend the workspace, thus it does not need too many links as the manipulator. However, until now, more researches have been done to investigate the dynamics of mobile manipulator with the fullactuated joints (Tan \& Xi 2002, Yamamoto \& Fukuda 2002, Jamisola \& Ang 2002) and robot underactuated manipulators (De Luca 2000). And less research is made about the topic of the mobile underactuated manipulator. The system consists of kinematic constraints (nonholonomic mobile base) which geometrically restrict the direction of mobility, and dynamic constraints (second order nonholonomic constraint) due to dynamic balance at passive degrees of freedom where no force or torque is applied. Therefore, the authors proposed several control methods for nonholonomic mobile manipulator when its hybrid joints are underactuated.

\subsection{Dynamics}

Considering $n$ DOF redundant manipulator with the hybrid joints mounted on a nonholonomic mobile base and supposing the trajectories of the links are constrained in the horizontal plane, then the links' gravity $G(q)=0$. The vector of generalized coordinates $q$ may be separated into two sets $q=\left[\begin{array}{ll}q_{v} & q_{r}\end{array}\right]$, where $q_{v} \in R^{m}$ describes the vector of generalized coordinates appearing in the constraints, and $q_{r} \in R^{k}$ are free vector of generalized coordinates; $n=m+k$. Therefore, the kinematic constraints can be simplified:

$$
A_{v}\left(q_{v}\right) \dot{q}_{v}=0
$$

with the constraint matrix $A_{v}\left(q_{v}\right) \in R^{r \times m}$. Then, the model is:

$$
\begin{gathered}
{\left[\begin{array}{ll}
M_{11} & M_{12} \\
M_{21} & M_{22}
\end{array}\right]\left[\begin{array}{l}
\ddot{q}_{v} \\
\ddot{q}_{r}
\end{array}\right]+\left[\begin{array}{ll}
C_{11} & C_{12} \\
C_{21} & C_{22}
\end{array}\right]\left[\begin{array}{l}
\dot{q}_{v} \\
\dot{q}_{r}
\end{array}\right]+\left[\begin{array}{c}
A_{v}^{T}\left(q_{v}\right) \lambda \\
0
\end{array}\right]=\left[\begin{array}{c}
E_{v} \tau_{v} \\
T \tau_{r}
\end{array}\right]+\left[\begin{array}{c}
0 \\
J^{T} F
\end{array}\right]} \\
q_{r}=\left[\begin{array}{c}
q_{a(i-1) \times a(i-1)} \\
q_{p} \\
q_{a(k-i) \times a(k-i)}
\end{array}\right], T=\left[\begin{array}{ccc}
E_{(i-1) \times(i-1)} & \\
0 &
\end{array}\right],(0 \leq i \leq k)
\end{gathered}
$$

where $[M] \in R^{n \times n}$ is the symmetric and positive definite inertia matrix; $[C] \in R^{n \times n}$ is the centrifugal and Coriolis force vector; $F$ represents the external force on the arm; $q_{p}$ and $q_{a}$ denote the vector of generalized coordinates of the passive joint and the active joint, respectively. A switching matrix $T \in R^{k \times k}$ corresponding to the $k$ hybrid joints is introduced here. This matrix $T$ is a diagonal matrix and the elements in the matrix are either 0 or 1 , if the element $T_{i i}=1$, the joint control is set to active mode; whereas it is set to passive mode if $T_{i i}=0 . \tau_{v} \in R^{m-r}$ represents the actuated torque vector of the constrained coordinates; $E_{v} \in R^{m \times(m-r)}$ represents the input transformation matrix, $\lambda \in R^{r}$ is the Lanrange multiplier.

\subsection{Recovery Control Design}

Supposing the arm has $n$ joints. If unexpected collision happens to the $i$ th link of the arm, the $i$ th joint is turned into the passive mode, these joints from the $(i+1)$ th joint to the $n$th joint are blocked. The system has controllable (i-1) joints after collision. If the left active DOF (the number 
of active joints) of system is more than the DOF of workspace, the manipulator is still redundant. Therefore the arm could be reconfigured to recover the end-effector's position. Otherwise, the mobile base and the arm are cooperated to compensate the end-effector's displacement.

\subsubsection{Recovery Control Using Arm}

If the manipulator is still redundant after collision, that is, the left active joints in the horizontal plane is more than or equal to 2 , we may configurate manipulator to compensate the passive motion of the $i$ th link. The blocked link rotates the $i$ th joint to attenuate the impact force and realize the force following. After the collision, the position of the endeffector $\left(x_{\text {effector, }} y_{\text {effector }}\right)$ can be obtained as:

$$
\left[\begin{array}{l}
x_{\text {effector }} \\
y_{\text {effector }}
\end{array}\right]=\left[\begin{array}{l}
x_{i} \\
y_{i}
\end{array}\right]+\left[\begin{array}{l}
l_{i} \cos \left(\theta_{i}+\Delta \theta\right) \\
l_{i} \sin \left(\theta_{i}+\Delta \theta\right)
\end{array}\right]+\sum_{j=i+1}^{n}\left[\begin{array}{l}
l_{j} \cos \phi_{j} \\
l_{j} \sin \phi_{j}
\end{array}\right]
$$

where $\left(x_{i}, y_{i}\right)$ is the initial position of the $i$ th joint in the global frame. $\theta_{i}$ is the angle of the $i$ th link relative to the global frame before the collision. $\Delta \theta$ is displacement angle of the contact force exerting on the $i$ th link relative to the global. $\phi_{j}$ is the joint angle in the joint workspace.

All the blocked links could be regards as one link; the coordinates for the equivalent mass center $G$ and orientation of the link are expressed as:

$$
\left[\begin{array}{l}
x_{G} \\
y_{G}
\end{array}\right]=\frac{1}{\sum_{j=i}^{n} m_{j}}\left[\begin{array}{l}
\sum_{j=i}^{n} m_{j} x_{j} \\
\sum_{j=i}^{n} m_{j} y_{j}
\end{array}\right]
$$

where $\left(x_{j}, y_{j}\right)$ is the coordinate of mass center of link $j$ and $\theta_{G}$ is the operational angle of the equivalent mass center $G$ in the global frame, $m_{j}$ is the mass of link $j$. The $P$ point is called as the center of percussion, which can be obtained as:

$$
K=\left(\sum_{j=i}^{n} I_{j}+\sum_{j=i}^{n} m_{j}\left(x_{j}^{2}+y_{j}^{2}\right)\right) / \sum_{j=i}^{n} m_{i} \cdot \sqrt{x_{G}^{2}+y_{G}^{2}}
$$

where $I_{j}$ is the inertia moment of link $j$.

To compensate the position displacement, a dynamic feedback linearization control is adopted (De Luca A. and Oriolo G., 2000). Define the $P$ point Cartesian position $\left(x_{p}, y_{p}\right)$ as:

$$
\left[\begin{array}{l}
x_{p} \\
y_{p}
\end{array}\right]=\left[\begin{array}{l}
x_{i} \\
y_{i}
\end{array}\right]+K\left[\begin{array}{l}
c \theta_{G} \\
s \theta_{G}
\end{array}\right]
$$

where $c \theta=\cos \theta, s \theta=\sin \theta$.

Let $\left[\ddot{x}_{i} \quad \ddot{y}_{i}\right]^{T}=R\left(\theta_{G}\right)\left[\begin{array}{ll}\xi & \sigma_{2}\end{array}\right]^{T}, \dot{\xi}=\eta, \dot{\eta}=\sigma_{1}, R\left(\theta_{G}\right)$ is the rotation matrix, $\xi$ is the linear acceleration of the $P$ point along the line OP. $\sigma_{2}$ is the linear acceleration of the base of the line $O P$ along the normal to its line. Therefore, we obtain as:

$$
\left[\begin{array}{c}
\sigma_{1} \\
\sigma_{2}
\end{array}\right]=\left[\begin{array}{cc}
1 & 0 \\
0 & \frac{K}{K \dot{\theta}_{G}^{2}-\xi}
\end{array}\right]\left(R^{T}\left(\theta_{G}\right)\left[\begin{array}{c}
x_{p}^{[4]} \\
y_{p}^{[4]}
\end{array}\right]-\left[\begin{array}{c}
\left(K \dot{\theta}_{G}{ }^{2}-\xi\right) \dot{\theta}_{G}^{2} \\
2 \eta \theta_{G}
\end{array}\right]\right)
$$




$$
\begin{gathered}
\xi=\ddot{x}_{p} c \theta_{G}+\ddot{y}_{p} s \theta_{G}+K \dot{\theta}_{G}^{2} \\
\dot{\theta}_{G}=\frac{y_{p}{ }^{[3]} c \theta_{G}-x_{p}{ }^{[3]} s \theta_{G}}{\ddot{y}_{p} s \theta_{G}+\ddot{x}_{p} c \theta_{G}} \\
\eta=\dddot{x}_{p} c \theta_{G}+\dddot{y}_{p} s \theta_{G}
\end{gathered}
$$

The initial position and the displacement of end effector are known, utilizing the above equations, the end effector could be returned to the desired position with the desired velocity during the time $T$. The inverse kinematics of the manipulator is used to obtain the position and velocity of the base of the jth joint $(j<i)$ :

$$
q_{r j}^{d}=f_{a}^{-1}\left(x_{i}, y_{i}, \theta_{i}\right)
$$

where $f_{a}^{-1}$ is the inverse kinematics of the manipulator from the ith joint to 1 st joint, $q_{r j}^{d}$ provide the reference trajectory to jth joint of the manipulator to position of the 1st joint at the desired position. The $q_{r}^{d}$ will provide the reference trajectory to each actuated joint of the arm to the desired position after collision disturbance. An actuator command for the robot arm $\tau_{a}$ can be obtained by using a suitable feedback control system according to (7) and (17) as follows:

$$
\tau_{a}=M_{22}\left(\ddot{q}_{r}^{d}+K_{a v}\left(\dot{q}_{r}^{d}-\dot{q}_{r}\right)+K_{a p}\left(q_{r}^{d}-q_{r}\right)\right)+C_{22}
$$

where $K_{a p} \in R^{n a \times n a}$ and $K_{a v} \in R^{n a \times n a}$ are the positions during an unexpected collision with the moving object and the velocity gain matrices, respectively ; $\dot{q}_{r}^{d}$ and $\ddot{q}_{r}^{d}$ are derived numerically by first and second differences of the desired joint angles $q_{r}^{d}$,respectively.

\subsubsection{Recovery Control Using Mobile Base}

If the number of the arm's active joints meets the following equation:

$$
n_{\text {active }}<n_{\text {workspace }}-1
$$

where $n_{\text {active }}$ is the number of the arm's active joints, $n_{\text {workspace }}$ is the DOF of the workspace. De Luca, Oriolo (2000) solved the problem of the controllability of trajectory to the desired state from any initial state, however, the robot had at least two active joints, i.e., the arm's join number $n \geq 3$ (as descriptions in 3.2.1). Therefore, in this chapter, we will consider the case of the join number $n<3$ ( that is, $n=1$ or $n=2$ ) and the arm has one underactuted joint. The number of the arm's active joints ( the control inputs) is less than that of workspace. By the mobile base, however, under the nonholonomic constraint, the methods of position control are proposed using the cooperation of the mobile base and the arm to realized controllability of trajectory to the desired position from an initial state.

Assume the motion is constrained in $2 D$ environment; then the whole arm is underactuated and demonstrated in Fig. 3, where $O$ is the mass centre of the mobile base; the joint $O$ is $(x$, $y$ ) in the base frame; $O A$ is $d ; \varphi$ is the direction angle of the mobile base. 


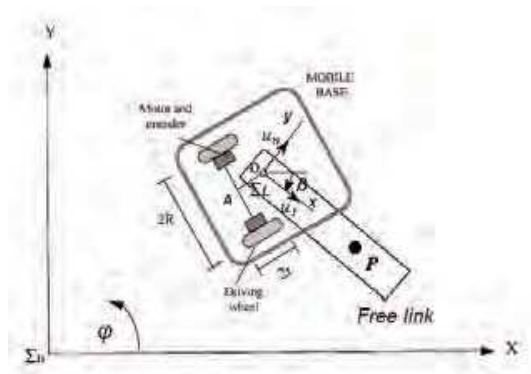

Fig. 3. Mobile manipulator with one free link.

The nonholonomic constraint states that the robot can only move in the direction normal to the axis of the driving wheels, i.e., the mobile base satisfies the conditions of pure rolling and the no slipping. Two motion controllers of the translational controller and the rotational controllers extended from the works of Arai and Tanie (1998) are proposed here for controlling the free link. But the difference from Arai and Tanie(1998) is that the motion of the free link is realized with the nonholonomic mobile base.

The nonholonomic constraint can be represented with the state vector $q_{v}=\left[\begin{array}{lll}x & y & \varphi\end{array}\right]^{T}$ and the input velocity $v=\left[\begin{array}{ll}v_{c} & \omega_{c}\end{array}\right]^{T}$ in the $C$ space as following:

$$
\left[\begin{array}{c}
\dot{x} \\
\dot{y} \\
\dot{\varphi}
\end{array}\right]=\left[\begin{array}{cc}
\cos \varphi & d \sin \varphi \\
\sin \varphi & d \cos \varphi \\
0 & 1
\end{array}\right]\left[\begin{array}{l}
v_{c} \\
\omega_{c}
\end{array}\right]
$$

The new input variables $\left(u_{t}, u_{n}\right)$ for the joint of the free link, that is, the center $O$ in Fig. 3, could be expressed as:

$$
\left[\begin{array}{l}
u_{t} \\
u_{n}
\end{array}\right]=\left[\begin{array}{cc}
\sin (\varphi-\beta) & \cos (\varphi-\beta) \\
-\cos (\varphi-\beta) & \sin (\varphi-\beta)
\end{array}\right]\left[\begin{array}{c}
a_{\perp O A} \\
a_{O A}
\end{array}\right]
$$

where $u_{t}$ is the acceleration component in the $\mathrm{x}$ direction of $\sum L$, and the component, $u_{n}$, normal to it. The vector $\left(a_{O A}, a_{\perp O A}\right)$, which represents one acceleration sub-vector along the line $O A$ and another normal to it, could be transformed from (20) as:

$$
\left[\begin{array}{c}
a_{\perp O A} \\
a_{O A}
\end{array}\right]=\left[\begin{array}{c}
d \dot{\omega}_{c} \\
\dot{v}_{c}
\end{array}\right]+\left[\begin{array}{c}
0 \\
-\omega_{c}^{2} d
\end{array}\right]
$$

And then

$$
\left[\begin{array}{c}
a_{\perp O A} \\
a_{O A}
\end{array}\right]=\left[\begin{array}{c}
\left(a_{L}+a_{R}\right) / 2-d \cdot\left(v_{R}-v_{L}\right)^{2} / 4 R^{2} \\
d \cdot\left(a_{R}-a_{L}\right) / 2 R
\end{array}\right]
$$

where $v_{L}, a_{L}$ and $v_{R}, a_{R}$ are the velocities and accelerations of the left wheel and right wheel, respectively.

From (21-23), we can get, 


$$
\left[\begin{array}{l}
a_{L} \\
a_{R}
\end{array}\right]=\left[\begin{array}{ll}
c(\varphi-\beta)-\frac{R}{d} \cdot s(\varphi-\beta) & s(\varphi-\beta)+\frac{R}{d} c(\varphi-\beta) \\
c(\varphi-\beta)+\frac{R}{d} \cdot s(\varphi-\beta) & s(\varphi-\beta)-\frac{R}{d} c(\varphi-\beta)
\end{array}\right]\left[\begin{array}{l}
u_{t} \\
u_{n}
\end{array}\right]+d\left[\begin{array}{l}
\frac{\left(v_{R}-v_{L}\right)^{2}}{4 R^{2}} \\
\frac{\left(v_{R}-v_{L}\right)^{2}}{4 R^{2}}
\end{array}\right]
$$

The coordinates of $P$ point $\left(x_{p}, y_{p}\right)$, that is, the center of percussion $P$, can be represented by the inputs $\left(a_{L}, a_{R}\right)$ of the mobile base as:

$$
\left.\left[\begin{array}{c}
\ddot{x}_{p} \\
\ddot{y}_{p} \\
\ddot{\beta}
\end{array}\right]=\left[\begin{array}{cc}
\cos \beta & 0 \\
\sin \beta & 0 \\
0 & 1 / k
\end{array}\right]\left[\begin{array}{cc}
c(\varphi-\beta)-\frac{R}{d} s(\varphi-\beta) & s(\varphi-\beta)+\frac{R}{d} c(\varphi-\beta) \\
c(\varphi-\beta)+\frac{R}{d} s(\varphi-\beta) & s(\varphi-\beta)-\frac{R}{d} c(\varphi-\beta)
\end{array}\right]^{-1}\left[\begin{array}{c}
a_{L} \\
a_{R}
\end{array}\right]-d\left[\begin{array}{l}
\frac{\left(v_{R}-v_{L}\right)^{2}}{4 R^{2}} \\
\frac{\left(v_{R}-v_{L}\right)^{2}}{4 R^{2}}
\end{array}\right]\right)
$$

Then it can be transformed from as

$$
\ddot{\beta}=u_{n} / K ; \ddot{x}_{p}=u_{t} \cos \beta ; \ddot{y}_{p}=u_{t} \sin \beta
$$

Assuming the free link is translation motion along the $x$-coordinates of $\sum B$, and the initial and desired configuration of $\mathrm{P}$ point at $\mathrm{x}$-axis are $x_{0}$ and $x_{e}$. We first plan the trajectory of the $P$ point, which is composed of the acceleration and deceleration procedure:

$$
\left\{\begin{array} { l } 
{ \ddot { x } _ { d } = a _ { 1 } } \\
{ \dot { x } _ { d } = a _ { 1 } t } \\
{ x _ { d } = x _ { 0 } + \frac { 1 } { 2 } a _ { 1 } t ^ { 2 } }
\end{array} \quad \left\{\begin{array}{l}
\ddot{x}_{d}=a_{2} \\
\dot{x}_{d}=a_{2}(t-T) \\
x_{d}=x_{e}+\frac{1}{2} a_{2}(t-T)^{2}
\end{array}\right.\right.
$$

for $(0 \leq t<T / 2)$ and $(T / 2 \leq t<T)$ respectively, and $T$ is the time period of the trajectory segment:

$$
\left[\begin{array}{l}
a_{1} \\
a_{2}
\end{array}\right]=\left[\begin{array}{c}
4\left(x_{e}-x_{0}\right) / T^{2} \\
-4\left(x_{e}-x_{0}\right) / T^{2}
\end{array}\right] \text { when } \begin{gathered}
0<t<T / 2 \\
T / 2<t<T
\end{gathered}
$$

where $x_{d}$ denotes the instaneous reference trajectory, then $x_{p}$ is the actual trajectory:

$$
u_{t}=\left\lfloor\ddot{x}_{d}+k_{v} e_{v}+k_{p} e_{p}\right\rfloor / \cos \beta
$$

where $e_{v}=\dot{x}_{d}-\dot{x}_{p}$ and $e_{p}=x_{d}-x_{p}$. And using the following to eliminate the error of yp and $\beta$ :

$$
u_{n}=-2 k \dot{\beta} \tan \beta+k \cos ^{2} \beta \cdot v / a_{i}(i=1,2)
$$

where $v$ is the new input. The converted system is:

$$
\frac{d}{d t}\left[\begin{array}{c}
y_{p} \\
\dot{y}_{p} \\
z_{1} \\
z_{2}
\end{array}\right]=\left[\begin{array}{llll}
0 & 1 & 0 & 0 \\
0 & 0 & 1 & 0 \\
0 & 0 & 0 & 1 \\
0 & 0 & 0 & 0
\end{array}\right]\left[\begin{array}{c}
y_{p} \\
\dot{y}_{p} \\
z_{1} \\
z_{2}
\end{array}\right]+\left[\begin{array}{l}
0 \\
0 \\
0 \\
1
\end{array}\right] v
$$

where $z_{1}=a_{i} \tan \beta, z_{2}=\dot{z}_{1}$

$$
v=k_{1} y_{p}+k_{2} \dot{y}_{p}+k_{3} z_{1}+k_{4} z_{2}
$$


when the input $v$ is transformed to the input $u_{n}$ by (29), $y_{p}$ and $\beta$ are stabilized to zero.

If the free link is in rotational motion, the input $u_{n}$ is given to turn the orientation of the link to follow the desired trajectory:

$$
u_{n}=k\left[\ddot{\beta}_{d}+k_{v} e_{v}+k_{p} e_{p}\right]
$$

where $\beta_{d}$ denotes the reference of $\beta$ on the trajectory, $e_{v}=\dot{\beta}_{r e f}-\dot{\beta}$ and $e_{p}=\beta_{\text {ref }}-\beta$.

The $P$ point position error $\left(e_{x}, e_{y}\right)$ in the base frame can be represented using $\left(e_{t}, e_{n}\right)$ in the link frame $\sum L$ :

$$
\left[\begin{array}{l}
e_{t} \\
e_{n}
\end{array}\right]=\left[\begin{array}{cc}
\cos \beta & \sin \beta \\
\sin \beta & -\cos \beta
\end{array}\right]\left[\begin{array}{l}
e_{x} \\
e_{y}
\end{array}\right]
$$

Integrating $\ddot{e}_{x}=-u_{t} \cos \beta, \ddot{e}_{y}=-u_{t} \sin \beta$, we get from (33),

$$
\frac{d}{d t}\left[\begin{array}{c}
e_{t} \\
e_{n} \\
\dot{e}_{t} \\
\dot{e}_{n}
\end{array}\right]=\left[\begin{array}{cccc}
0 & 0 & 1 & 0 \\
0 & 0 & 0 & 1 \\
\dot{\beta}^{2} & -\ddot{\beta} & 0 & -2 \dot{\beta} \\
\ddot{\beta} & \dot{\beta}^{2} & 2 \dot{\beta} & 0
\end{array}\right]\left[\begin{array}{c}
e_{t} \\
e_{n} \\
\dot{e}_{t} \\
\dot{e}_{n}
\end{array}\right]+\left[\begin{array}{c}
0 \\
0 \\
-1 \\
0
\end{array}\right] u_{t}
$$

when the link rotates with constant velocity $\dot{\beta}>0$, the input $u_{t}$ is substituted as $\tau=\dot{\beta} t$, $u_{t}=\dot{\beta}^{2} v,(34)$ can be expressed as

$$
\frac{d e_{\dot{\beta}}}{d t}=\left[\begin{array}{cccc}
0 & 0 & 1 & 0 \\
0 & 0 & 0 & 1 \\
1 & 0 & 0 & -2 \\
0 & 1 & 2 & 0
\end{array}\right] e_{\dot{\beta}}+\left[\begin{array}{c}
0 \\
0 \\
-1 \\
0
\end{array}\right] v
$$

where $e_{\dot{\beta}}=\left[\begin{array}{llll}e_{t} & e_{n} & d e_{t} / d \tau & d e_{n} / d \tau\end{array}\right]^{T}$. The system can be controlled by a linear state feedback. If $\dot{\beta}<0$, then $\tau=-\dot{\beta} t$ is applied.

When the angular acceleration is considered, and the $\dot{\beta}$ is negligible, if $\ddot{\beta}>0$, the input $u_{t}$ is substituted as $\tau=\sqrt{\ddot{\beta}} t, u_{t}=\ddot{\beta} v,(34)$ becomes

$$
\frac{d e_{\ddot{\beta}}}{d t}=\left[\begin{array}{cccc}
0 & 0 & 1 & 0 \\
0 & 0 & 0 & 1 \\
0 & -1 & 0 & 0 \\
1 & 0 & 0 & 0
\end{array}\right] \cdot e_{\ddot{\beta}}+\left[\begin{array}{c}
0 \\
0 \\
-1 \\
0
\end{array}\right] \cdot v
$$

where $e_{\ddot{\beta}}=\left[\begin{array}{llll}e_{t} & e_{n} & d e_{t} / d \tau & d e_{n} / d \tau\end{array}\right]^{T}$. The system can be controlled by a linear state

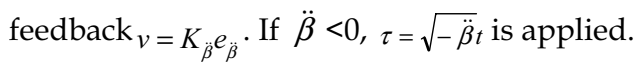

Give the initial position and the goal position, if the free link has an initial angular velocity, the free arm could be rest with the rotation controller by the input $\left(a_{L}, a_{R}\right)$ of mobile base from (32), (35), (36) and then rotate the free link until it is in alignment with the desired position, and then mobile base pushes the free link to the desired position using translation controller by the input $\left(a_{L}, a_{R}\right)$ from (28), (29), (30). 
Develop Human Safety Mechanism for

\subsubsection{Motion Control Using Virtual Link}

If the number of the arm's active joints meets the following equation:

$$
n_{\text {active }}=n_{\text {workspace }}-1
$$

where $n_{\text {active }}$ is the number of the arm's active joints, $n_{\text {workspace }}$ is the DOF of the workspace. The arm has one underactuated link as shown in Fig.4.

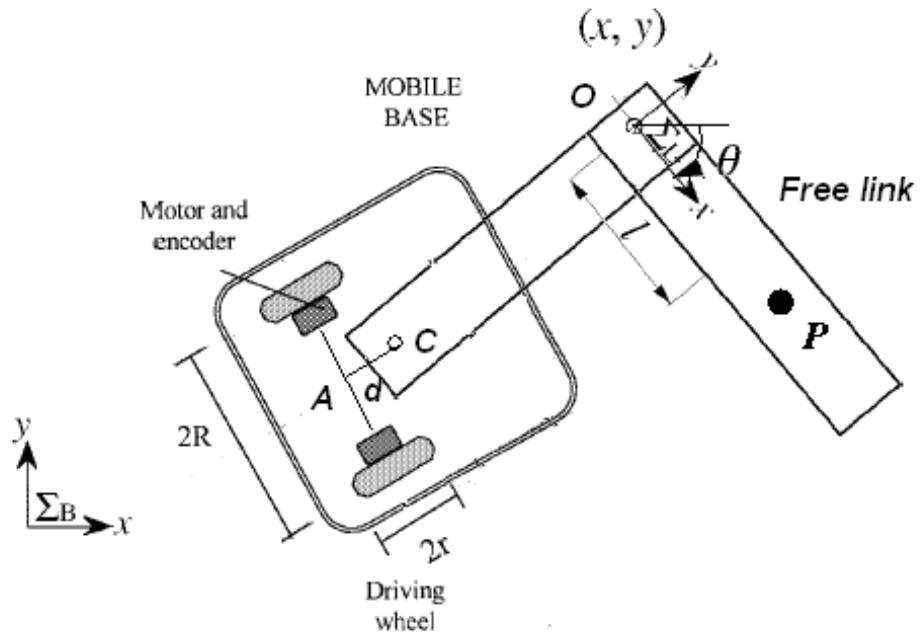

Fig. 4. Mobile manipulator with one free link.

In this case, we utilize the cooperation of both arm and mobile base to move the end-effector to the desired position. The locomotion of the mobile base could augments the DOF of the system, but the mobile base is nonholonomic, which makes the mobile base only moving in the specified direction, rather than arbitrary direction. We have to plan the specified trajectory to cope with the nonholonomic constraint. To solve this problem, we propose a method applying a virtual link fixed on the mobile base.

Two cases are discussed here. If the desired position is in the workspace of the manipulator, that is, the position can be reached by the rotation of the base and the active link. We treat line $C A$ as a virtual link in Fig. 4. The rotation of mobile base around $A$ is equivalent to one revolve joint.

To track the desired position, we make full use of the virtual link $C A$ and the active link $C O$. At first, we could get the $P$ point position $\left(x_{p}, y_{p}\right)$ from $(10)$ and $O$ point position $(x, y)$. The control method as same as (11) - (17) are used to complete the position control, and an actuator command $\tau_{m}=\left[\begin{array}{ll}\tau_{A} & \tau_{C}\end{array}\right]^{T}$ for the robot system can be obtained by a suitable feedback control system according to (7) as follows:

$$
\tau_{m}=M\left(\ddot{q}_{C, A}^{d}+K_{a v}\left(\dot{q}_{C, A}^{d}-\dot{q}\right)+K_{a p}\left(q_{C, A}^{d}-q\right)\right)+C
$$

where $M_{a} \in R^{3 \times 3}$ is the symmetric inertia submatrix of the arm which include the virtual link. $C \in R^{2 \times 1}$ is the centrifugal and Coriolis force vector of the arm. For the virtual joint, $\left(\tau_{L} / r+\tau_{R} / r\right) R=\tau_{A}$ and $\tau_{L}=-\tau_{R} \cdot K_{a p}$ and $K_{a v}$ are the position gain parameter and the velocity gain parameter, respectively; $\dot{q}_{C, A}^{d}$ and $\ddot{q}_{C, A}^{d}$ are derived numerically by first and second 
differences of the desired joint angles respectively. Using this control, the end-effector trajectory tracking can be realized despite the motion of the passive link.

If the desired position is in the workspace of the mobile manipulator, the mobile base has to move, we assume the mobile base is substituted with a virtual link, whose length could be equal to that of the free link and which augments the DOF of the system, and the link is connected to the joint $C$ of the arm with a virtual joint. The trajectory of virtual joint could be regarded as the motion of the mobile base. Therefore, the DOF of the whole system is equal to the DOF of the workspace. This status of the system is similar with Section 3.2.1. Then, we can adopt the same control algorithm as the above section. If the mobile base can track the trajectory of the joint, the control can be realized. But we must first plan the trajectory of the mobile base from the initial position to the position of the virtual joint. Therefore, there exists a control problem how to drive the mobile under underactuated manipulator.

Based on the translational and rotational controllers for the free link in Section 3.2.2, the free link probably has an initial rotation velocity because of switching, the input $\left(u_{t}, u_{n}\right)$ of active joint $C$ from (28), (29) is utilized to eliminate this angular velocity to rest the free link. Then we recover $\theta$ to the desired position (Although $\theta$ is changed in eliminating angular velocity of free link). Then, we adopt the above controllers (32), (35), (36) and (28), (29) to complete rotation or translation motion of free link by the mobile base and the left active links. When the mobile base reaches the desired point, we regulate the posture of the mobile base to $\theta \pm \pi / 2$. The second step adopts the (11)-(17) to complete the motion control.

\section{Simulation and Experiment Studies}

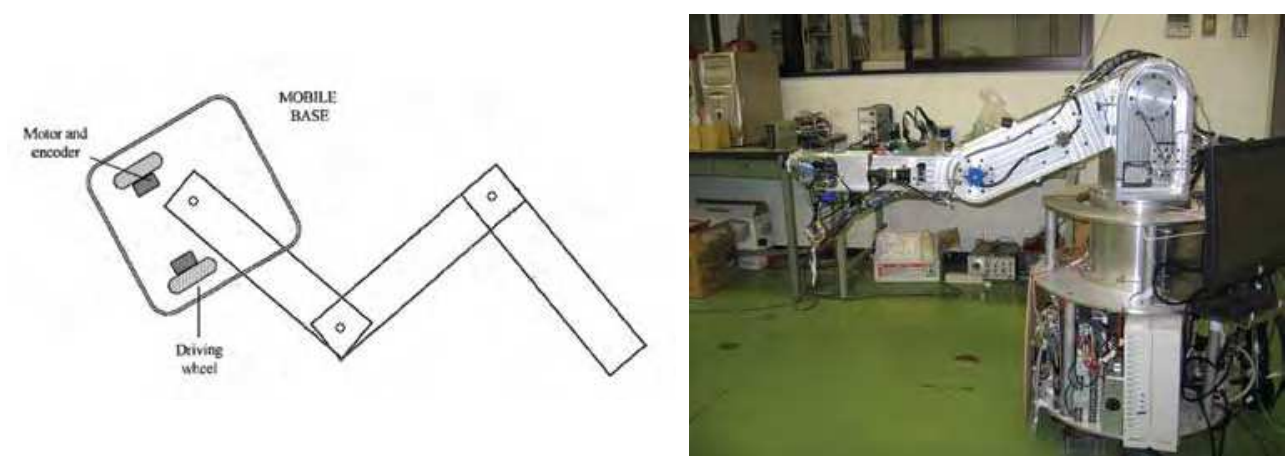

Fig. 5. (a) Simulation of three link planar robot (left); (b) Hybrid joint based mobile manipulator (right).

\subsection{Hybrid Joint Response Experiment}

The rapid response of hybrid joint, that is, the time of switching modes, is an important impact factor to achieve the safety for both robot and human, therefore, a shorter time of switching hybrid joint would greatly improve the safety of system. Therefore, we conduct the tests to measure the switching time of the hybrid joint as shown in Fig. 1. The status of hybrid joint is switched from the active mode to passive mode. The releasing time is shown in the Fig.6. The average response time is about $0.045 \mathrm{~s}$, if we assume the impact velocity is $0.5 \mathrm{~m} / \mathrm{s}$, the deformation of link's surface would be $0.0225 \mathrm{~m}$. Therefore, it is better that the thickness of soft covering should be more than $0.05 \sim 0.06 \mathrm{~m}$ (considering the response time of emergency stop). 


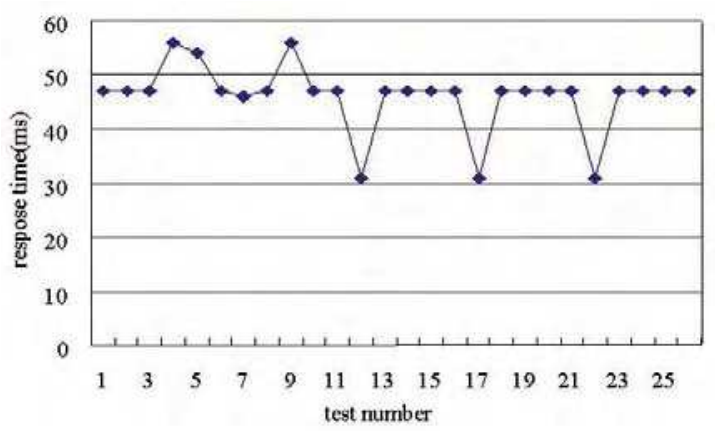

Fig. 6. The switching time of hybrid joint.

\subsection{Impact Simulation}

Assuming we adopt the static/dynamic thresholds of the human pain tolerance limit parameterized by the contact force $F_{\text {limit }}=50 \mathrm{~N}$ in the reference of Yamada's criterion (Yamada, Suita, etc., 1997), and the arm is safe only if the impact velocity is less than about $0.25 \mathrm{~m} / \mathrm{sec}$ in the paper (Yamada, Suita, etc., 1997). We execute the impact simulation with a 3-DOF planar twowheeled mobile manipulator using the hybrid joints in the Fig. 5 (a). Assume a human collides with the last link. The parameters of the mobile manipulator is $m_{1}=3 \mathrm{~kg} ; m_{2}=3 \mathrm{~kg} ; m_{3}=5 \mathrm{~kg} ; l_{1}=1.5 \mathrm{~m}$; $l_{2}=1 \mathrm{~m} ; l_{3}=1 \mathrm{~m} ; k=2 / 3 \mathrm{~m}$. The stiffness and damping of the arm covered with the soft materials and the human are selected as $k=800 \mathrm{~N}^{-1} \mathrm{~m}^{-1}, c=100 \mathrm{~N} . \mathrm{s} . \mathrm{m}^{-1}$. The human's mass is $50 \mathrm{~kg}$, and the maximum impulse velocity of the obstacle could reach to $0.5 \mathrm{~m} / \mathrm{s}$ under the $F_{\text {limit }}=50 \mathrm{~N}$.The evolutions of the impact forces on the different collision points of last link are shown in Fig. 7. We can see that the impact forces on difference collision points are attenuated quickly. Compared with the results of Lim, Tanie $(1999,2000)$ and Yoon, Kang, etc. (2003), under the condition of no damage to the human, the impact velocity has been improved and the impact forces have been decreased. When the collision happens to other links, the inertia of moment is increased; however, in the case of hybrid joints, the impact force is dependent on coefficients $k$ and $c$, which can be different values on the different links to meet the limit of impulse force.

\subsection{Impact Experiments and Discussions}

The impact experiments have been done using the hybrid joints in mobile manipulator as shown Fig. 5(b). Two joints in the horizontal plane are hybrid type and are used to make experiments.

As similar as the simulations, the arm is with the hybrid joint and covered with a soft

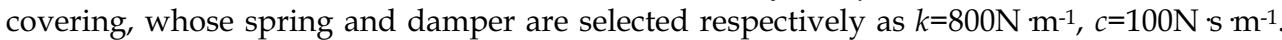
To confirm our objectives for simplicity, the impact experiments are conducted using: 1) the rigid arm without the hybrid joint ; 2) The rigid arm with the hybrid joint.

Experiment 1: The human collisions with the last link with the soft covering but without hybrid joint, the human moves in approximately constant velocity $0.5 \mathrm{~m} / \mathrm{s}$. The collision point on the link is $0.3 \mathrm{~m}$ from the joint. The parameters of the collision link of the arm is $M=5 \mathrm{~kg}$ (the fifth link), $L=0.3 \mathrm{~m}$ (the fifth's length), and the collision point is $0.3 \mathrm{~m}$ to the joint. The contact forces are measured with the force/torque sensor attached to the contacted link of the arm.

Experiment 2: The experiment condition is similar with experiment 1, but the fifth joint equipped with hybrid joint of the arm causes an unexpected collision with the human in approximately constant velocity $0.5 \mathrm{~m} / \mathrm{s}$. 


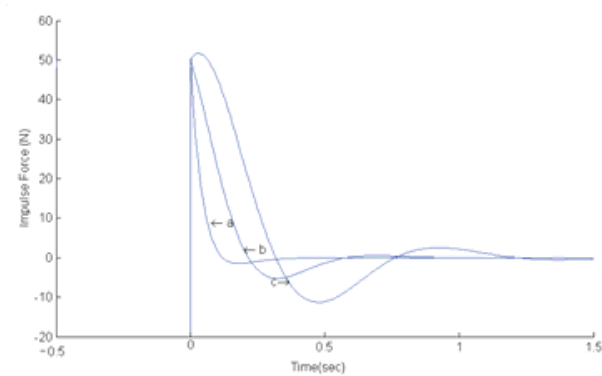

Fig. 7. The profile of contact force (blue line) when object collisions with third link, (a): collision point is $0.3 \mathrm{~m}$ relative to the passive joint; $(\mathrm{b})$ : collision point is $0.5 \mathrm{~m}$ relative to the passive joint ; (c):collision point is $0.7 \mathrm{~m}$ relative to the passive joint. The human veolcity is $0.5 \mathrm{~m} / \mathrm{s}$.
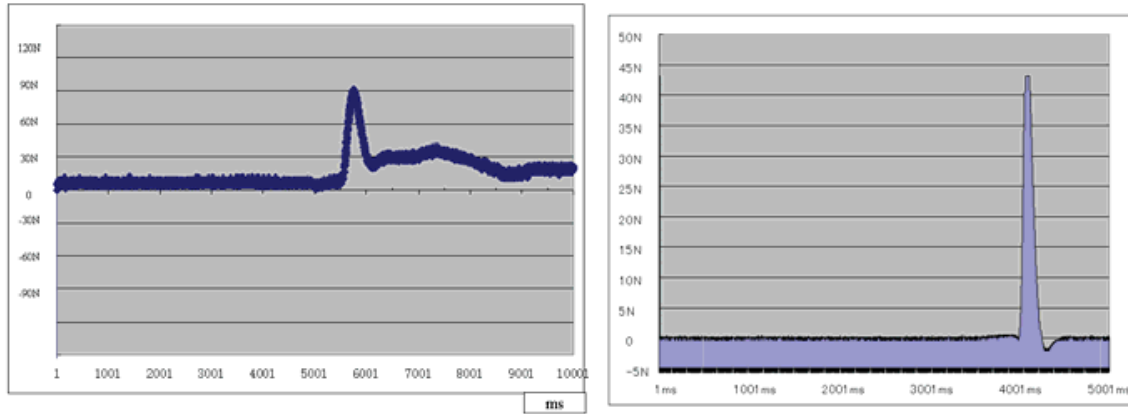

Fig. 8. (a) Contact force of the experiment with soft covering but without hybrid joint (left); (b) Contact force of the experiment with soft covering and hybrid joint (right).

In the experiment 1 , for the rigid arm with the soft covering but without hybrid joint, the contact force is extremely large, as shown in Fig. 8(a), which is similar as Fig.15 (a) in Lim, Tanie (1999). However, the duration time of the impact force more than $50 \mathrm{~N}$ is approximately $0.4 \mathrm{~s}$ and it is attenuated slowly, because without the fast complaint deformation of link (or the deformation takes place relatively slowly), the inertia of human makes both objects contacted. It is apparent that it would make damage to the human.

For the experiment 2 , the link has an initial velocity about $0.2 \mathrm{rad} / \mathrm{s}$. When the collision takes place, the trigger on the link is contacted, the system commands the emergency stop and then the circuit releases the hybrid joint, the response of collision force is shown in Fig. 8(b), which shows that the contact force is remarkably attenuated, damped more quickly and lasts shorter time compared with the previous works of Lim, Tanie (1999) and Yoon, Kang, etc. (2003). But the collision experiment result is a little different from the simulations because they are executed in the ideal situation where no external disturbances (including the friction and unmodeled dynamics, etc) have been considered. Therefore, the hardware experiments prove that mechanism is effective.

\subsection{Recovery Control Simulations}

To thoroughly verify the proposed methods, the recovery control simulations are presented for every possible H-R interaction case. In Simulation I, the mobile manipulator is shown in Fig.4 (a), the collision happens to 3rd link, then, the end-effector moves from initial position $(1.49 \mathrm{~m}, 1.67 \mathrm{~m})$ to another position $(1.3163 \mathrm{~m}, 1.9708 \mathrm{~m})$, and the third joint angle changes 
from 400 to 200 with the angular velocity $(0.47 \mathrm{rad} / \mathrm{s})$. We have to recover the task position as soon as the collision link separates with human. Our intention is to drive the third joint to the position $(0.5503 \mathrm{~m}, 1.3280 \mathrm{~m}, 200)$ so that the end-effector goes back to the initial position. Since the system is still redundant, we only adopt the recovery control using the arm from (11)-(18). The trajectories of the end-effector and the third joint are shown in Fig.9 (a). The position errors of end-effector are shown Fig. 9(b). From these two figures, we can see the actual trajectory of the end-effector has converged to the desired position.

In Simulation II, we assume a human collides with the second link, the second joint angle change from $40 \mathrm{deg}$ to $20 \mathrm{deg}$, the third joint angle is $90 \mathrm{deg}$ and the third joint becomes blocked. The second joint is switched to passive mode. And then, we adopt the recovering control using a virtual link. We can treat the third and the second link as one link and the equivalent mass center $G$ relative to the second joint is $0.7905 \mathrm{~m}$. Therefore, we can calculate $K=1.0541 \mathrm{~m}$. The end-effector's initial position is $(2.5 \mathrm{~m}, 2.5 \mathrm{~m})$ with the angular velocity $0.5 \mathrm{rad} / \mathrm{s}$, the goal position is $(1.9546 \mathrm{~m}, 1.1671 \mathrm{~m})$. The angular velocity has been first eliminated and the desired angle of passive link has been obtained before the regulation process. The control result is shown in Fig.11. Then the regulation process of the mobile base to the virtual joint position is shown in Fig.12. And the errors of ycoordinate of $P$ point and ${ }_{\beta}$ in the regulation course are shown in Fig. 13. Then, recovery control is going on. Because the third joint is a blocked joint, we utilize the position feedback controller as the same as the Simulation I and the position trajectories of the end-effector and 2nd joint are shown in Fig.10. Within 20s, the end-effector recovers the initial position. During the recovering, the trajectory of the mobile base is shown in Fig. 12. At last, the error evolution of the end-effector position is shown in Fig.14. From the figures, we know the end-effector converge to the desired position.
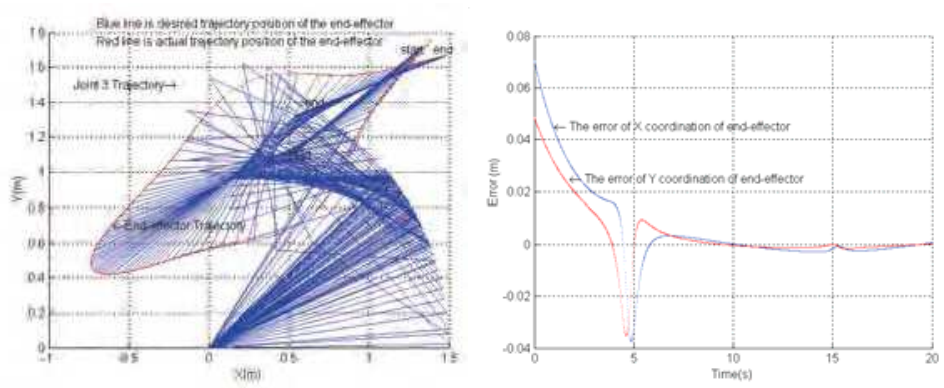

Fig. 9. (a)The trajectory of the end-effector and the third joint position; (b) Displacement of end-effector.

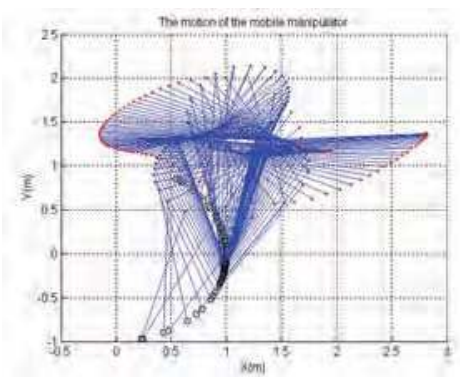

Fig. 10. The actual position trajectory of the mobile manipulator, the circles represent the base, the blue points denote the 2 nd joint, and the red points denote the end-effecotr, $3 \mathrm{rd}$ joint is the blocked and is not shown. 


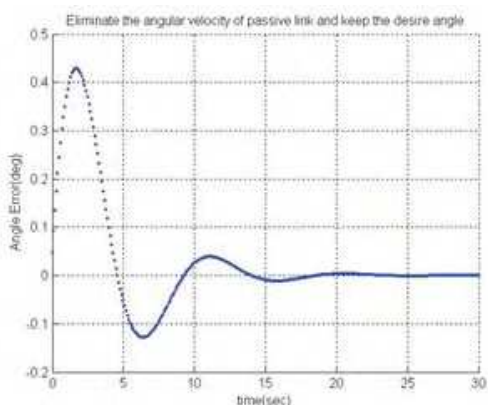

Fig. 11. Eliminate the angular velocity casued by the collision and keeping the angle of passive joint to the desired angle before the recovery control. In this case,the desired angle of passive joint is 200 before the recovery.

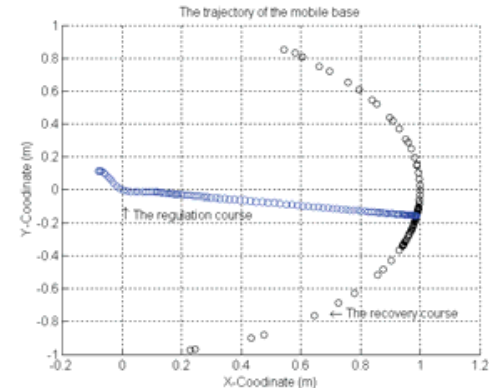

Fig. 12. The trajectory of the mobile base.

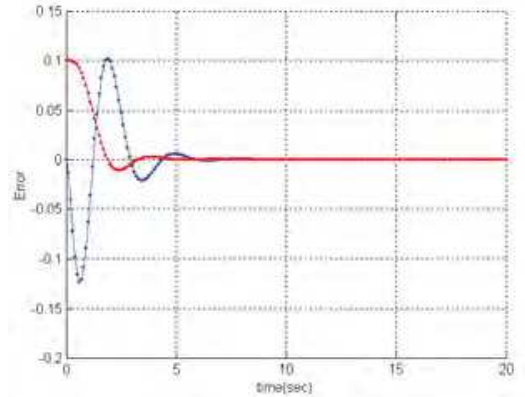

Fig. 13. Error of $y$ (red line) and ${ }_{\beta}$ (blue line)in the regulation course.

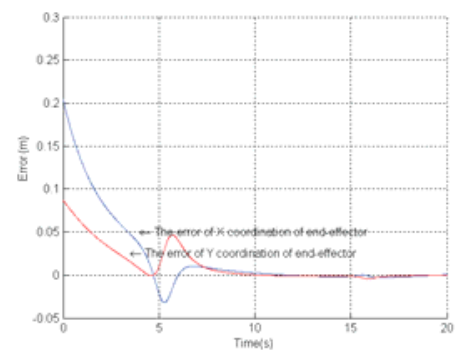

Fig. 14. Displacement of the end-effector. 
Develop Human Safety Mechanism for

\subsection{Recovery Control Experiments}

For the mobile manipulator we make experiments as shown in Fig.5 (b), we use the mobile base to realize the recover control. The length of the free link is $0.3 \mathrm{~m}$ and its mass is $5 \mathrm{~kg}, k=0.185 \mathrm{~m}$. The free joint is $87.2 \mathrm{~cm}$ relative to the center of the mobile base. The initial Angle $0.1351 \mathrm{rad}$ with zero velocity, the desired goal angle is $0 \mathrm{rad}$. On the time $t=1.7 \mathrm{~s}$, an impact force is put on the link, and then the link is with an angular velocity. The rotation angle and the angular velocity of free link under control are shown in the Fig.15, and after 20s, it approaches the desired angle. The trajectory of the mobile base is shown in Fig.16 and the final position is $(4.155 \mathrm{~cm},-1.1126 \mathrm{~cm})$. Then, the mobile base turns around the center, and makes the direction angle align with the free link. The translation of free link with the mobile base goes on. The expected motion of the free link is to translate the passive link along the $y$-axis from $(0 \mathrm{~cm}, 87.2 \mathrm{~cm}$,$) to the position (0 \mathrm{~cm}, 127.2 \mathrm{~cm})$, but the initial position of the passive joint is $\left(9.47 \mathrm{~cm}, 87.2 \mathrm{~cm}, 0^{\circ}\right)$, that is, we need to eliminate the $x$ coordinate error to $0 \mathrm{~cm}$ and keep the displacement angle of the free link to $0 \mathrm{rad}$. The both control errors results are shown in Fig.17 and Fig.18. And the corresponding trajectory of the mobile base is demonstrated in Fig. 19. Due to the movement error of the mobile base, the position of the mobile base only arrives the $(-0.219 \mathrm{~cm}, 40.6 \mathrm{~cm})$, but the end-effector has arrived the position ($\left.0.219 \mathrm{~cm}, 127.8 \mathrm{~cm}, 0^{\circ}\right)$ in the tolerant error.

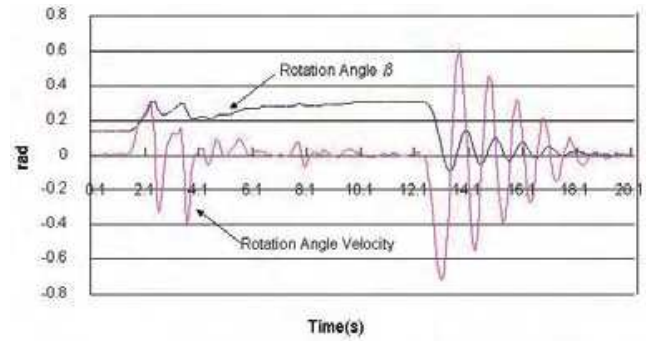

Fig. 15. The rotation of passive link, the desired angle $\beta$ is 0 rad.

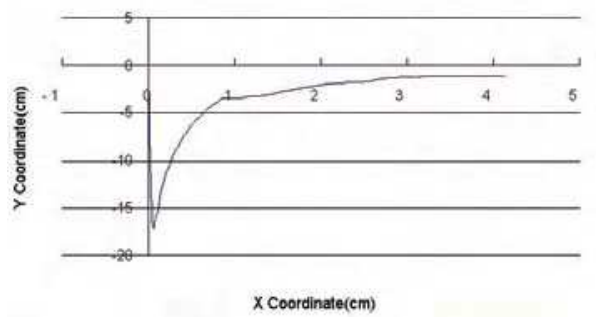

Fig. 16. The trajectory of mobile base for the rotation $\beta$.

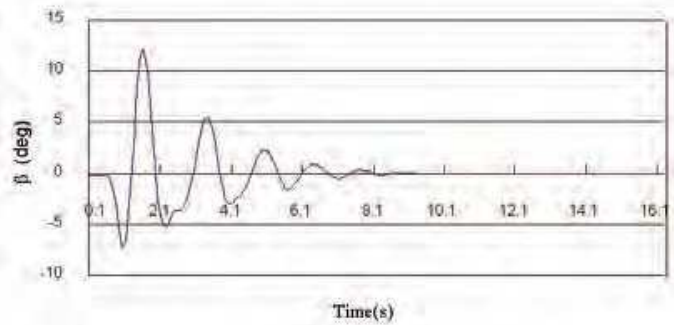

Fig. 17. Angle of the passive link by the mobile base, the desired $\beta$ is $0 \mathrm{rad}$. 


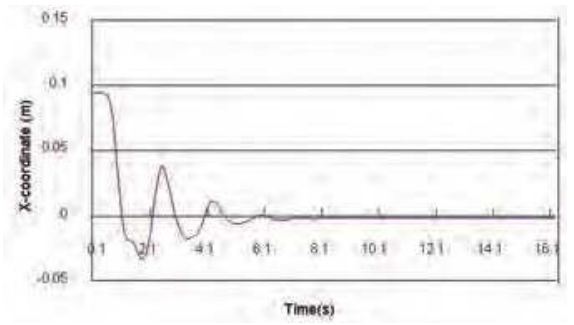

Fig. 18. Error of the passive joint, the desired $x$ coordinate is $0 \mathrm{~m}$.

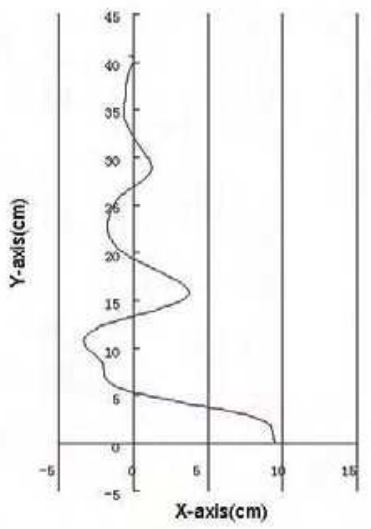

Fig. 19. The trajectory of mobile base for the translation.

\section{Conclusions}

A hybrid joint scheme has been suggested in order to assure the safe interaction in human-robot cooperative tasks. During an unexpected collision between the robot and human, the suppression of collision/contact forces has been investigated using the hybrid joints, and the control laws for the restoration of desired position have also been developed. This control laws are capable of maneuvering the end-effector position, which is tolerant to unexpected contact forces with the environment. The joint configurations are re-adjusted according to the position of the end-effector to keep performing the given task. The mobile base is utilized to improve the flexibility of the system. This kind of system can be implemented easily and employed effectively in unknown dynamic environments such as offices, and home environments, due to the demonstrated capabilities of compliance behavior and collision tolerance. Validity of the proposed mechanism and control strategy has been verified by simulation and experimental tests.

\section{References}

Anderson, R. J. \& Spong, M. W. (1987). Hybrid impedance control of robotic manipulators, Proc. IEEE Int. Conf. Robotics and Automation, pp. 1073-1080.

Arai, H. ; Tanie, K.\& Shiroma, N. (1998). Nonholonomic Control of a Three-DOF Planar Underactuated Manipulator, IEEE Tran.on Robotics and Automation, Vol. 14, No. 5, pp. 681-695. 
Cutkosky, M. R. \& Wright, P. (1986). Active control of a compliant wrist in manipulating task, ASME J. Engineering for Industry Vol. 108, pp. 36-43.

De Luca, A. \& Oriolo, G. (2000). Motion planning and trajectory control of an underactuated three-link robot via dynamic feedback linearization, Proc. IEEE International Conference on Robotics and Automation, pp. 2789-2795.

Fugie, M. G. ; Tani, T. ; \& Hirano, K. (1994). Walking rehabilitation system for the elderly, Proc. IEEE/RSJ Int. Conf. Intelligent Robots and Systems, pp. 1655-1662.

Goldenberg, A. A. (1987) orce and impedance control of robot manipulators, IEEE Trans. Robotics and Automation, Vol. 4, No. 6, pp. 653-660.

Goswami, A. \& Peshkin, M. A. (1993). Task-space/joint-space damping transformations for passive redundant manipulators, Proc. IEEE Int. Conf. Robotics and Automation, pp. 642-647.

Kawamura, K. \& Iskarous, M. (1994 ). Trends in service robots for the disabled and the elderly, Proc. IEEE/RSJ Int. Conf. Intelligent Robots and Systems, pp. 1647-1653.

Kazerooni, K. H. ; \& Waibel, B. J. (1988). Theory and experiment on the stability of robot compliance control, Proc. IEEE Int. Conf. Robotics and Automation, pp. 71-87.

Laurin-Kovitz, K. F. ; Colgate, J. E. ; \& Carnes, S.D.R. (1991). Design of components for programmable passive impedance, Proc. IEEE Int. Conf. Robotics and Automation, pp. 1476-1481.

Lim, H. O. \& Tanie, K. (1999). Collision-tolerant control of human-friendly robot with viscoelastic trunk. IEEE/ASME Trans. Mechatronics, Vol. 4, No. 4., pp. 417-427.

Lim, H. \& Tanie, K. (2000). Human Safety Mechanisms of Human-Friendly Robots: Passive Viscoelastic Trunk and Passively Movable Base, The International Journal of Robotics Research vol. 19, No. 4, pp. 307-335.

Lindsay, T. S. ;Sinha, P. R. ; \& Paul, R. P. (1993). An instrumented compliant wrist for robotics applications. Proc. IEEE Int. Conf. Robotics and Automation, pp. 648-653.

Li, Z. ; Ming, A. ; Xi, N. ; Shimojo, M. \& Kajitani, M. (2004) .Mobile Manipulator Collision Control With Hybrid Joints in Human-robot Symbiotic Environments,Proc. IEEE/RSJ International Conference on Intelligent Robots and Systems, pp. $154-161$

Jamisola, R.; Ang, M.H., Jr.; Oetomo, D.; Khatib, O.; Tao Ming Lim; Ser Yong Lim (2002). The Operational Space Formulation implementation to aircraft canopy polishing using a mobile manipulator, IEEE International Conference on Robotics and Automation, pp. $400-405$

Mills, J. K. (1990). Stability of robotic manipulators during transition to and from compliant motion, Automatica, Vol. 26, No. 5, pp. 861-874

Morita, T., \& Sugano, S. (1995). Design and development of a new robot joint using a mechanical impedance adjuster, Proc. IEEE Int. Conf. Robotics and Automation, pp. 2469-2475.

Morita, T., \& Sugano, S. (1996).Development of 4-d.o.f. manipulator using mechanical impedance adjuster, Proc. IEEE Int. Conf. Robotics and Automation, pp. 2902-2907.

Morita, T., Shibuya, K., and Sugano, S. (1998). Design and control of mobile manipulation system for human symbiotic humanoid: Hadaly-2, Proc. IEEE Int. Conf. Robotics and Automation, pp. 1315-1320.

Raibert, M. H., and Craig, J. J. (1981). Hybrid position/force control of manipulator. ASME J. Dynamics Systems, Measurement, and Control, Vol. 102, No. 2, pp. 126-133.

Salisbury, J. K. (1980). Active stiffness control of a manipulator in Cartesian coordinates. Proc. IEEE Int. Conf. Decision and Control, pp. 91-97. 
Schutter, J. D., \& Van Brussel, H. (1988). Compliant robot motion I: A formalism for specifying compliant motion tasks, Int. J. Robotics Research, Vol. 7, No. 4, pp. 3-17.

Suita, K. ; Yamada, Y. ; Tsuchida, N. ; Imai, K. ; Ikeda, H. ; \& Sugimoto, N. (1995) . A failuretosafety "kyozon" system with simple contact with detection and stop capabilities for safe human-autonomous robot coexistence, Proc. IEEE Int. Conf. Robotics and Automation, pp. 3089-3096.

Tan, J. \& Xi, N. (2002) . Integrated task planning and control for mobile manipulators, IEEE Int. Conf. on Robotics and Automation, pp.382 - 387

Wedel, D. L. \& Saridis, G. N. (1988) .An experiment in hybrid position/force control of a six dof revolute manipulator. Proc. IEEE Int. Conf. Robotics and Automation, pp. 16381642.

Whitney, D. E. (1982). Quasi-static assembly of compliantly supported parts, ASME J. Dynamics Systems, Measurement, and Control, Vol. 104, No. 1, pp. 65-77.

Yamamoto, Y.; Fukuda, S. (2002). Trajectory planning of multiple mobile manipulators with collision avoidance capability, IEEE Int. Conf. on Robotics and Automation, pp. 3565 3570 .

Yamada, Y. ; Suita, K. ; Imai, K. ; Ikeda, H. \& Sugimoto, N.(1997). Human-robot contact in the safeguarding space, IEEE/ASME Tran. on Mechatronics, Vol. 2, No. 4, pp. 230236.

Yoon S. ; Kang S. ; Kim S. ;Kim Y. ;Kim M. ; Lee C. (2003). Safe Arm with MR-based Passive Compliant Joints and Visco-elastic Covering for Service Robot Applications, Proc. IEEE/RSJ Intl. Conference on Intelligent Robots and Systems, pp. 2191-2196. 


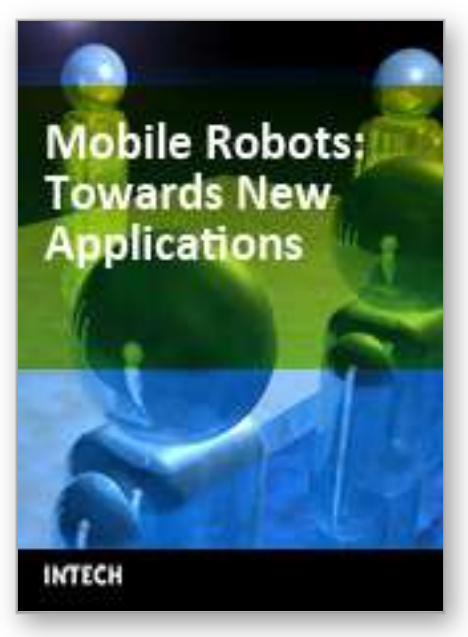

\author{
Mobile Robots: towards New Applications \\ Edited by Aleksandar Lazinica
}

ISBN 978-3-86611-314-5

Hard cover, 600 pages

Publisher I-Tech Education and Publishing

Published online 01, December, 2006

Published in print edition December, 2006

The range of potential applications for mobile robots is enormous. It includes agricultural robotics applications, routine material transport in factories, warehouses, office buildings and hospitals, indoor and outdoor security patrols, inventory verification, hazardous material handling, hazardous site cleanup, underwater applications, and numerous military applications. This book is the result of inspirations and contributions from many researchers worldwide. It presents a collection of wide range research results of robotics scientific community. Various aspects of current research in new robotics research areas and disciplines are explored and discussed. It is divided in three main parts covering different research areas: Humanoid Robots, Human-Robot Interaction, and Special Applications. We hope that you will find a lot of useful information in this book, which will help you in performing your research or fire your interests to start performing research in some of the cutting edge research fields mentioned in the book.

\title{
How to reference
}

In order to correctly reference this scholarly work, feel free to copy and paste the following:

Zhijun Li, Jun Luo, Shaorong Xie and Jiangong Gu (2006). Develop Human Safety Mechanism for HumanSymbiotic Mobile Manipulators: Compliant Hybrid Joints, Mobile Robots: towards New Applications, Aleksandar Lazinica (Ed.), ISBN: 978-3-86611-314-5, InTech, Available from:

http://www.intechopen.com/books/mobile_robots_towards_new_applications/develop_human_safety_mechani sm_for_human-symbiotic_mobile_manipulators_compliant_hybrid_joints

\section{INTECH}

open science | open minds

\author{
InTech Europe \\ University Campus STeP Ri \\ Slavka Krautzeka 83/A \\ 51000 Rijeka, Croatia \\ Phone: +385 (51) 770447 \\ Fax: +385 (51) 686166 \\ www.intechopen.com
}

\author{
InTech China \\ Unit 405, Office Block, Hotel Equatorial Shanghai \\ No.65, Yan An Road (West), Shanghai, 200040, China \\ 中国上海市延安西路65号上海国际贵都大饭店办公楼405单元 \\ Phone: +86-21-62489820 \\ Fax: +86-21-62489821
}


(C) 2006 The Author(s). Licensee IntechOpen. This chapter is distributed under the terms of the Creative Commons Attribution-NonCommercial-ShareAlike-3.0 License, which permits use, distribution and reproduction for non-commercial purposes, provided the original is properly cited and derivative works building on this content are distributed under the same license. 